\title{
Flow stress identification of tubular materials using the progressive inverse identification method
}

\author{
Erfan Asaadi ${ }^{*}$ and P. Stephan Heyns \\ Department of Mechanical and Aeronautical Engineering, University of Pretoria, Pretoria, South \\ Africa
}

\begin{abstract}
Purpose - Propose a progressive inverse identification algorithm to characterize flow stress of tubular materials from the material response, independent of choosing an a priori hardening constitutive model.

Design/methodology/approach - In contrast to the conventional forward flow stress identification methods, the flow stress is characterized by a multi-linear curve rather than a limited number of hardening model parameters. The proposed algorithm optimizes the slopes and lengths of the curve increments simultaneously. The objective of the optimization is that the finite element simulation response of the test estimates the material response within a predefined accuracy.
\end{abstract}

Findings - We employ the algorithm to identify flow stress of a 304 stainless steel tube in a tube bulge test as an example to illustrate application of the algorithm. Comparing response of the finite element simulation using the obtained flow stress with the material response shows that the method can accurately determine the flow stress of the tube.

Practical implications - The obtained flow stress can be employed for more accurate finite element simulation of the metal forming processes as the material behaviour can be characterized in a similar state of stress as the target metal forming process. Moreover, since there is no need for a priori choosing the hardening model, there is no risk for choosing an improper hardening model, which in turn facilitates solving the inverse problem.

Originality/value - The proposed algorithm is more efficient than the conventional inverse flow stress identification methods. In the latter, each attempt to select a more accurate hardening model, if it is available, result in constructing an entirely new inverse problem. However, this problem is avoided in the proposed algorithm.

\footnotetext{
* Corresponding author

Erfan Asaadi can be contacted at: er.asaadi@gmail.com Erfan Asaadi can be contacted at: er.asaadi@gmail.com
} 


\section{Keywords:}

Flow stress; inverse identification; hardening model; tube bulge test; metal forming; stressstrain identification

\section{Introduction}

Finite element methods (FEMs) are widely used to model and investigate various materials forming processes. The rising costs of the experimental investigations promote the use of numerical simulations to analyse these processes and to estimate the system parameters before finalising the detailed design. Hence, introducing well-evaluated material characteristics to the FEM is crucial for accurately simulating these processes and predicting the process parameters. Flow stress identification of tubular material is the focus of this paper. For brevity we sometimes refer to this as the stress-strain identification.

\subsection{Characterizing the stress-strain behaviour in multi-axial stress states}

Despite the seemingly simple implementation, uniaxial testing presents problems. The maximum obtainable strain in tensile tests before localized necking, and in uniaxial compression tests before buckling, is small in comparison with the maximum achievable strain during a forming process. In addition, (Zribi et al., 2013) considered the complexity of the stress state which governs the flow of material during forming, and showed that employing the stress-strain behaviour obtained in the uniaxial tests for the numerical simulation of metal forming processes may give rise to unacceptable discrepancies between the simulation results and the reality. It is therefore generally accepted that, to identify the stress-strain relation of the materials, experiments must be conducted to impose loads on an instrumented specimen, so that the resultant stress state is similar to the stress state that may be expected during the forming process.

For example, the results of a plain tensile test and a bulge test are combined to obtain the mechanical characteristics of sheet metals, suitable for simulating sheet metal forming (Aguir et al., 2011). This issue is even more noticeable if the results of a uniaxial tensile test are applied to simulate tube hydroforming, because the material is subjected to a biaxial or even triaxial stress state. Furthermore, the geometry of the tube makes it difficult to prepare the standard tensile testing samples, as flattening of the tubular materials imposes unwanted hardening to the samples before conducting the tests. This may cause discrepancies between the flow stress obtained for the flattened samples and the flow stress of the tubular material. Hence, a tube bulge test is usually accepted as the most appropriate experiment for measuring material behaviour for tube hydroforming simulations , e.g. see (Zribi et al., 2013, Koç et al., 2001, Nemat-Alla, 2003, Strano and Altan, 2004, Hwang et al., 2007, Bortot et al., 2008, Lianfa and Cheng, 2008, Xu et al., 2008, Hwang and Wang, 2009, Saboori et al., 2014). 
Several studies are reported in the literature concerning the determination of material properties for such multi-axial stress states. To position the work presented here, the evolution of the stress-plastic strain identification methods for tubes is first considered. It should however be realised that although the present work focuses on determining the flow stress of tubular materials, the proposed identification method could be generalized to be used in other tests, e.g. identifying the flow stress after necking in tensile test, sheet bulge test, etc.

(Koç et al., 2001) devised an analytical formulation for thin isotropic tubes subjected to a tube bulge test to convert the geometrical measurements of the bulged area and the applied pressure directly to stress-strain data. Subsequently (Nemat-Alla, 2003) proposed an inverse identification technique to characterize stress-strain behaviour in the hoop direction of tubular materials, by conducting a lateral compression test on the tube. The results of the finite element (FE) simulation of the process, based on an assumed material model, are compared to the experimental load-deflection curve in an iterative algorithm to minimize the error. The resultant material parameters determine the best predicted stress-strain curve for the assumed material model. In the same vein, (Strano and Altan, 2004) presented an inverse method for obtaining the stress-strain behaviour of tubular materials that are subjected to a tube bulge test. Their method is based on an energy balance approach, which assumes cosine-like form functions for the inner and outer profiles of the bulged region, and three parameters of the assumed flow stress model capturing the material behaviour. The external work and the internal work of deformation are calculated. Finally, the material parameters are adjusted to minimize the difference between the external and the internal work. The obtained material parameters determine the best achievable stress-strain curve, based on the selected constitutive hardening model.

(Hwang et al., 2007) assumed an elliptical shape for the bulged region of the tube in a tube bulge test to obtain an analytical relationship between equivalent stress and equivalent strain at the selected point. In this method, the thickness and height of the bulge peak are measured as a function of the internal pressure during the test. Bortot et al. (Bortot et al., 2008) developed an analytical stress-strain relationship for thin-walled tubes in a tube bulge test. Their method requires measuring the instantaneous thickness, the longitudinal and circumferential radii of curvatures and the internal pressure. (Lianfa and Cheng, 2008) established an analytical formulation based on the plastic membrane theory and force equilibrium equations to obtain the stress-strain relationship from the curvature of the bulged area and the applied pressure. (Xu et al., 2008) introduced an adaptive inverse finite element method to determine the material hardening coefficient and the hardening exponent, two material parameters for the assumed material model. This method is based on comparing the predicted results of the finite element simulations, using a set of assumed material parameters, with the experimental result by evaluating how different material parameters affect the shape of the pressure-bulge height curve. The predicted parameters are adjusted until the simulation result is matched with the actual result. 
(Hwang and Wang, 2009) further developed the earlier work conducted by Hwang et al. (Hwang et al., 2007) by considering the effects of anisotropy. (Zribi et al., 2013) applied an inverse identification method to identify the flow stress parameters of anisotropic tubular materials. The anisotropy effect is measured by tensile tests. This method associates the Nelder Mead simplex optimisation algorithm with the FEM and experimental results. The bulge height at the peak of the dome versus the internal pressure is measured as the experimental data. The inverse method iteratively searches for the best material hardening parameters that minimize the gap between the response of the finite element simulation and the test measurement. In order to increase the probability of finding the global minimum, or an acceptable local minimum, the algorithm is restarted at random points.

Recently, (Saboori et al., 2014) employed digital image correlation to measure circumferential and longitudinal curvature of the bulge area. Then, by employing an analytical approach, the measured data are used to generate effective stress and effective strain. The obtained stress-strain data are then used to calibrate Swift and Hollomon hardening models.

It is clear from the above that one can classify the methods for characterizing the stressstrain behaviour of the materials into two groups:

\section{Direct stress-strain identification methods}

Using this approach, the material characteristics of tubular profiles are obtained by establishing the analytical relationships, which directly convert the experimental measurement of the tube bulge test to the stress-strain data. Although the final computation for acquiring the stress-strain curve, after obtaining the relationship between the measurement and the stress-strain data, is quite straightforward and fast, the major limitation of these approaches is that they are mostly based on some assumption about the geometry of the experiment, such as thin-walled structures or a specific shape of the bulged area. They also may require some responses of the material, like curvatures in different directions, or thickness of the tube during the test, which are not easy to determine. This may compromise the accuracy of the results.

\section{Inverse forward stress-strain identification methods}

The inverse forward identification procedure converts the observed measurements of the system to some material parameters by determining the model parameters that minimize the difference between a predicted response obtained by finite element simulation of the test process, and the experimental observation of the system behaviour. Employing FE analysis of the system eliminates the geometrical simplifying assumptions about the shape of the bulged area. A major challenge of this approach is that assuming a specific material model is essential. This was done in a number of recent inverse identification problems, e.g. see (Zribi et al., 2013, Germain et al., 2013, Li et al., 2014, Cooney et al., 2015, Kang et al., 2014, Sun et al., 2014, Bocciarelli et al., 2014) despite the material still being unknown. Moreover, the constitutive models for some materials , e.g. see (Luczynski et al., 2015), or 
for non-conventional conditions, e.g. see (Nguyen et al., 2013, Noh et al., 2014, Dureja et al., 2014), may not be readily available. Therefore, several trials must be conducted to identify the proper material constitutive model. Each trial with a new material model results in running a new inverse identification problem. In addition, excessive computational costs are incurred by using multi-parameter material models and robust optimization methods to solve the multi-variable inverse problems.

These issues justify using an improved strategy, partly to deal with the problem of selecting an a priori hardening model; and partly to utilize FE simulation of the instrumented test to deal with inaccurate assumptions and the difficult measurements required by the analytical stress-strain behaviour identification methods.

In this paper, we first introduce an algorithm for progressive inverse flow stress identification which is independent of the a priori selection of a hardening constitutive model. In addition, some techniques to reduce the computational cost of the process are introduced. Then we make use of the algorithm to identify the flow stress curve of a 304 stainless steel tube in a tube bulge test. The identified flow stress curve is used to simulate the tube bulge test. Comparing the simulation response with the measured response of the material shows the effectiveness of the method to predict the flow stress behaviour of the material.

\section{Fundamental concepts of the progressive inverse stress-strain curve identification approach}

Similar to the conventional material inverse identification methods, the algorithm comprises proper experimental testing with the appropriate finite element analysis and an optimization algorithm. However, the proposed procedure does not require a priori consideration of the material hardening model. Hence, it can easily be employed for identifying stress-strain curve of the materials with unknown constitutive hardening model. The output is a multilinear stress-strain behaviour rather than a limited number of coefficients for a specific material constitutive hardening model.

Unlike the conventional forward inverse identification method which determines the entire stress-strain behaviour by obtaining the material hardening constitutive parameters, the progressive inverse identification method determines the stress-strain behaviour incrementally. The response of the material in the test is divided into optimally determined increments, which are associated with linear increments in the estimated multi-linear stressstrain behaviour (see Figure 1 and Figure 2). The two increments, namely the increment in the material response due to an imposed load increment and the linear increment in the estimated stress-strain curve, are related. In other words, determining one of them identifies the other.

In order to identify each increment, two parameters must be efficiently identified, namely the load at the end of the response increment (e.g. point 2 in Figure 1) and the material 
hardening rate of the corresponding stress-strain increment, i.e. the slope of line 1'-2' (in Figure 2).

The algorithm commences with the identification of the hardening behaviour by estimating the initial yield point.. Then a multi-objective optimization algorithm determines the optimal first hardening rate, slope of the line 1'-2', and the first load increment, point 2 . The next yield point is determined as the maximum obtained simulated stress for the selected increment, point 2'. Fixing the yield point at the maximum achieved stress has the benefit that size and the slope of the next increment does not influence the estimated error of the last identified increment. The algorithm continues this incremental procedure to identify the multi-linear hardening model for the finite element simulation of the test which estimates the response of the material.

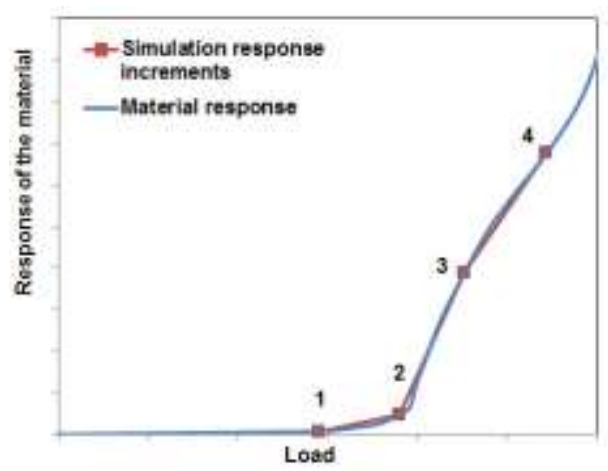

Figure 1. Typical response of the material, and the estimated simulation response.

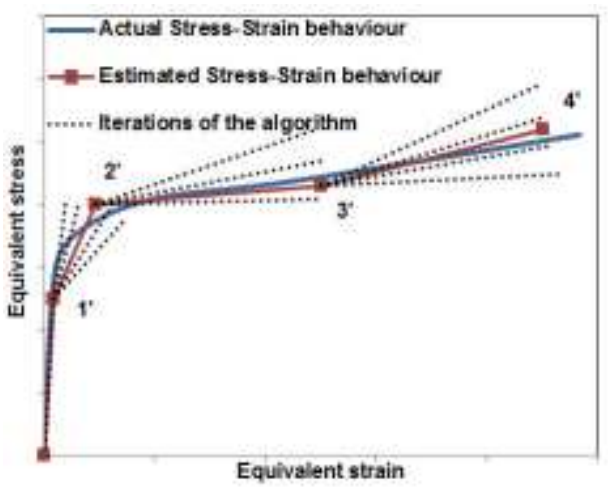

Figure 2. Actual and the estimated stress-strain curve.

In this work, we select the total displacement of the tube's bulge peak vs. internal hydraulic pressure, see Figure 3, as the response of the material being tested. (Zribi et al., 2013) and (Xu et al., 2008) already showed that the pressure-bulge height data contains enough information for constructing an inverse problem to identify hardening parameters of the material in a tube bulge test. Another objective function could be defined using, strain or displacement of more points on the bulge surface. However, we preferred to use the displacements of the other points on the surface for validating the results. 


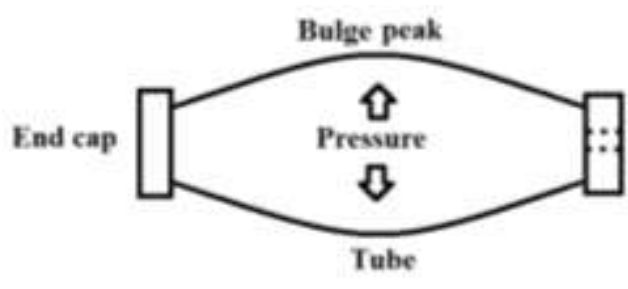

Figure 3. scheme of the bulged tube.

The progressive inverse flow stress identification method is now presented as a stepwise algorithm. The algorithm can be generalized easily for different applications; however for the sake of simplicity, we explain details of the algorithm for obtaining stress-strain behaviour of the tubular profiles in a tube bulge test.

1. Conduct an experiment to obtain the response of the instrumented tube to the imposed load. Figure 4 depicts the measured total displacement of the peak of the dome versus the hydraulic pressure (see red $+\mathrm{s}$ ). We refer to this as the response of the tubular material.

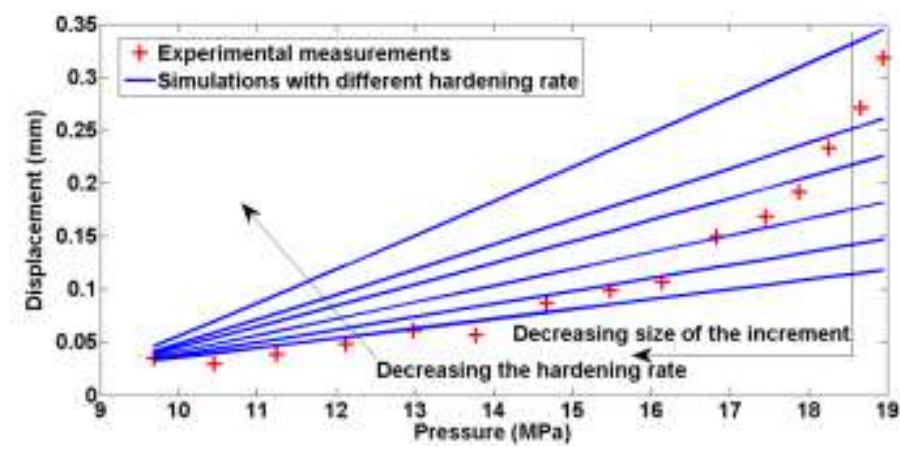

Figure 4. Responses of the material and the simulation responses, while the optimization algorithm iteratively changes the hardening rate.

2. Construct the FEM based on the experimental test conditions, e.g. FEM of the tube bulge test.

3. Assume the initial yield stress as the starting point, because we intend to just explore the plastic behaviour of the material. In this case, we estimate the yield point as the maximum simulated stress at the yield pressure. We define the yield pressure as the pressure that the simulation response, in which one assumes a linear elastic material, deviates from the material response.

4. Assume multi-linear hardening behaviour for the simulated material.

5. Define the first objective function which determines the error between the simulation response, and the response of the material as a function of the hardening rate and size of the load increment, e.g. error of the simulation for the known pressure and hardening rate.

6. Define the second objective function which determines the size of the load increment. 
7. Run the multi-objective optimization algorithm with the aim of determining the largest possible increment as well as the lowest estimated error. The outputs are the load increment size, and the hardening rate for the increment. As the increment size increases, the error of the simulation for the optimal hardening rate increases. This is due to the fact that the stress-strain behaviour of the material cannot be linearly estimated (see Figure 5). Therefore, the optimization results are a series of solutions; see Figure 6, called the Pareto front. For more information about the multi-objective optimizations see (Deb and Kalyanmoy, 2001). The larger the increments the larger the estimated error. Thus, the user has to make the decision of an acceptable estimation error for the given increment, which we refer to as the resolution.

8. For the selected hardening rate and the increment size, fix the yield point as the maximum equivalent stress in the simulation. Therefore, the increment is fully determined.

9. Go to step 4 to identify the next increment until the entire range of the material response is covered.

In order to clarify the procedure we determined the stress-strain behaviour associated with the response of the experiment, illustrated in Figure 5 with blue dots, assuming two different resolutions.

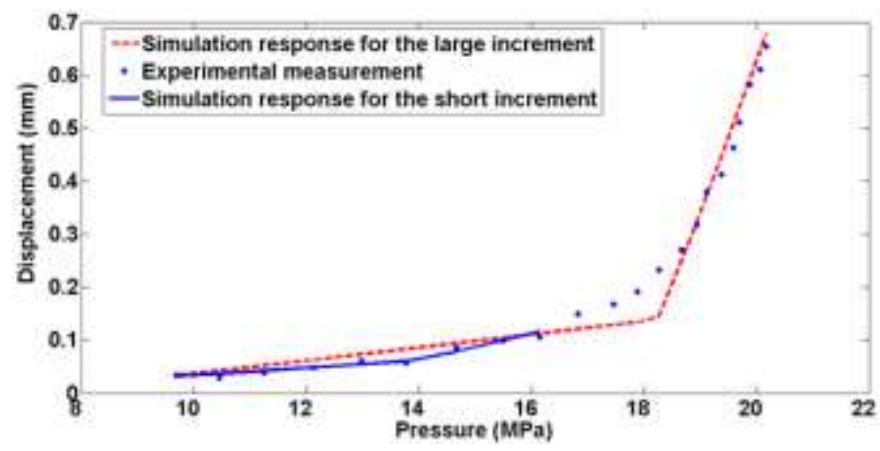

Figure 5. Material responses and the estimated material responses for the selected resolutions.

Here we employed a multi-optimization genetic algorithm to determine the set of the solutions for optimal load increment size and the hardening rate. We chose two solutions, one with a larger pressure increment and larger error as the lower resolution example, the red square, and the other with lower pressure increment and lower error as the higher resolution example, the blue circle in Figure 6. 


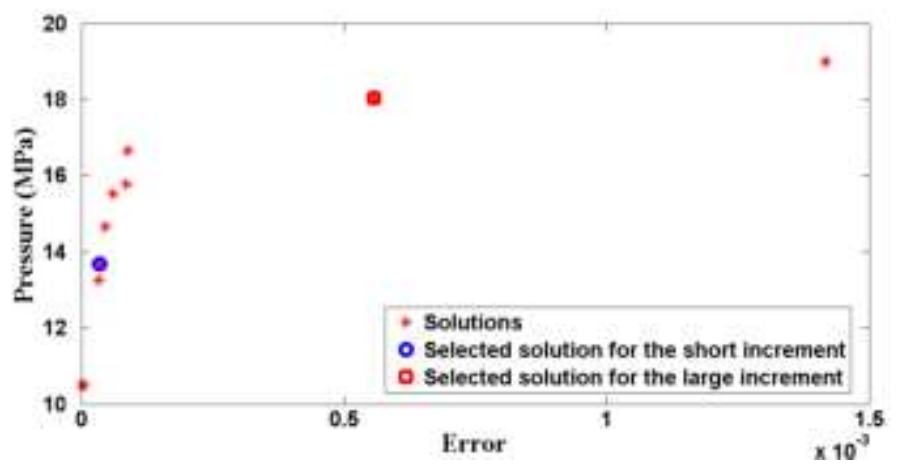

Figure 6. Solutions of the multi-objective optimization.

We solved the algorithm for the first two increments. The resultant linearly estimated stress-strain behaviours are illustrated in Figure 7, and the simulation responses using the obtained stress-strain behaviour are superimposed on the experimental results already depicted in Figure 5.

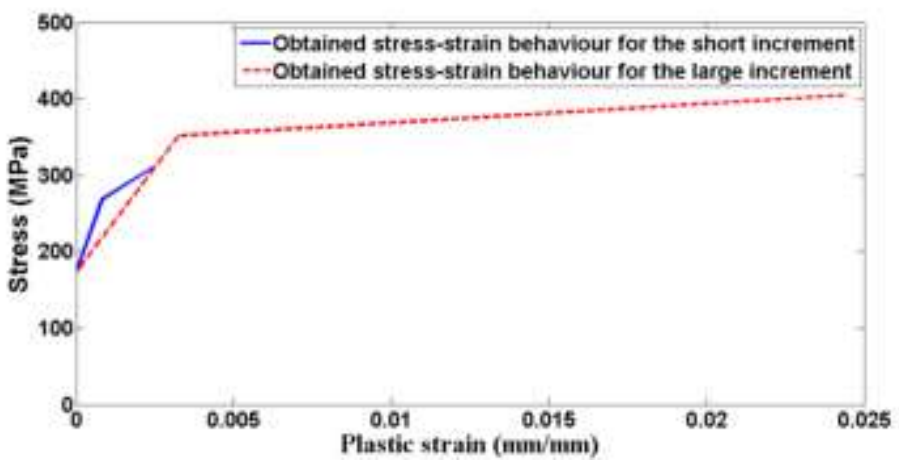

Figure 7. Estimated stress-strain behaviour for the selected resolutions.

Comparing the examples with higher and lower resolution shows how increasing the resolution and size of the increments represent a trade-off. The former increases the prediction accuracy, while the latter decreases the computational cost by estimating the material hardening behaviour with fewer linear increments.

In order to apply the progressive inverse identification algorithm for identifying material properties of the tubular material in the tube bulge test, the following observations are noteworthy:

- This method is used for characterizing the property of interest that is evolving with load, e.g. hardening behaviour. When considering other properties as well (such as friction and anisotropy) the relevant parameters describing these properties must be determined separately (e.g. see (Zribi et al., 2013)). Alternatively an inverse identification method which explores effects of the all parameters at the same time must be employed. However, the latter needs a priori assumption of the constitutive hardening model (e.g. see (Aguir et al., 2011, Prates et al., 2014, Bolzon and Talassi, 2013, Prates et al., 2015, Zhang et al., 2014)). 
- In the conventional forward inverse identification method, the suggested parameter sets for every iteration of the optimization algorithm must be simulated considering full load obtained by the experiment, e.g. point 4 in Figure 1. However, the stepwise nature of the progressive inverse identification method allows simulating each iteration with the pressure, lower than or equal to the maximum obtained load. This results in a lower computational cost.

- If the nature of the measurement error changes during the measurement, it is not always possible to set an efficient fixed allowable error for linearly estimating all of the increments. However, the obtained set of possible solutions always indicates the minimum achievable error in contrast with the size of the increment. Note that it is always advisable to select the measurement instrument based on the range of the parameters of the interest, e.g. using a low range pressure gauge for beginning of the tube bulge test and high range pressure gauge for the rest of the pressure measurement. Therefore, the measurement noise is more consistent through the entire range of the measurement.

- If the selected increments are too short, the method models the noise rather than the variation of the material properties.

- In order to apply the method to obtain the material hardening behaviour after instability, e.g. after occurrence of necking in uniaxial tensile test, we suggest using imposed displacement as the load and measured reaction force as the response of the material.

The aim of the next section of the paper is to demonstrate the application of the method to obtain the stress-strain curve of a 304 stainless steel tube by conducting the tube bulge test and applying the progressive inverse identification method to identify stress-strain behaviour of the tube.

\section{Stress-strain identification procedure for a 304 stainless steel tube}

\subsection{The tube bulge test}

\subsubsection{The experimental setup}

In order to conduct the tube bulge test, a $63.5 \mathrm{~mm} \times 1.4 \mathrm{~mm}$ laser-welded 304 stainless steel tube was selected. One application of the selected tube is hydroforming of exhaust manifolds in the automotive industry. Caps were welded to the ends of the sample tube. The lower cap was fixed to the test setup and the upper cap remained free (Figure 8 and Figure 9). 


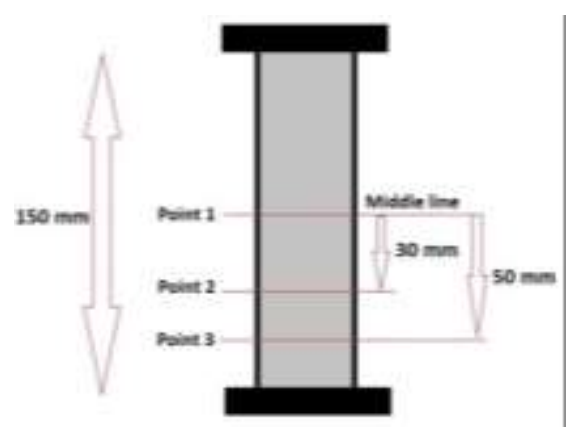

Figure 8. Schematic of test sample

Initially a tube bulge forming die and a hand pump were employed to define the boundary conditions of the test and to provide the pressure. However, high measurement noise caused by the friction between the tube and the die, and also the pressure fluctuations, necessitated a change in the setup.

Subsequently a single-stroke booster pump was devised to reduce the pressure fluctuations. The pump comprised a single-stroke cylinder squeezed between the rams of a servohydraulic universal testing machine. Since there is accurate velocity control on the actuator speed, the bulge test could be conducted at a constant flow rate. By eliminating the reciprocating motion inherent in the majority of positive-displacement hydraulic pumps, the pressure noise could be reduced considerably (Figure 9).

A constant feeding rate of $233 \mathrm{ml} / \mathrm{min}$ for the bulge forming was selected. The measurement at the points on the peak of the bulged area, point 1 in Figure 8 was used to determine the stress-strain curves, and the measurement of the other points along the length was used for validating the results. 


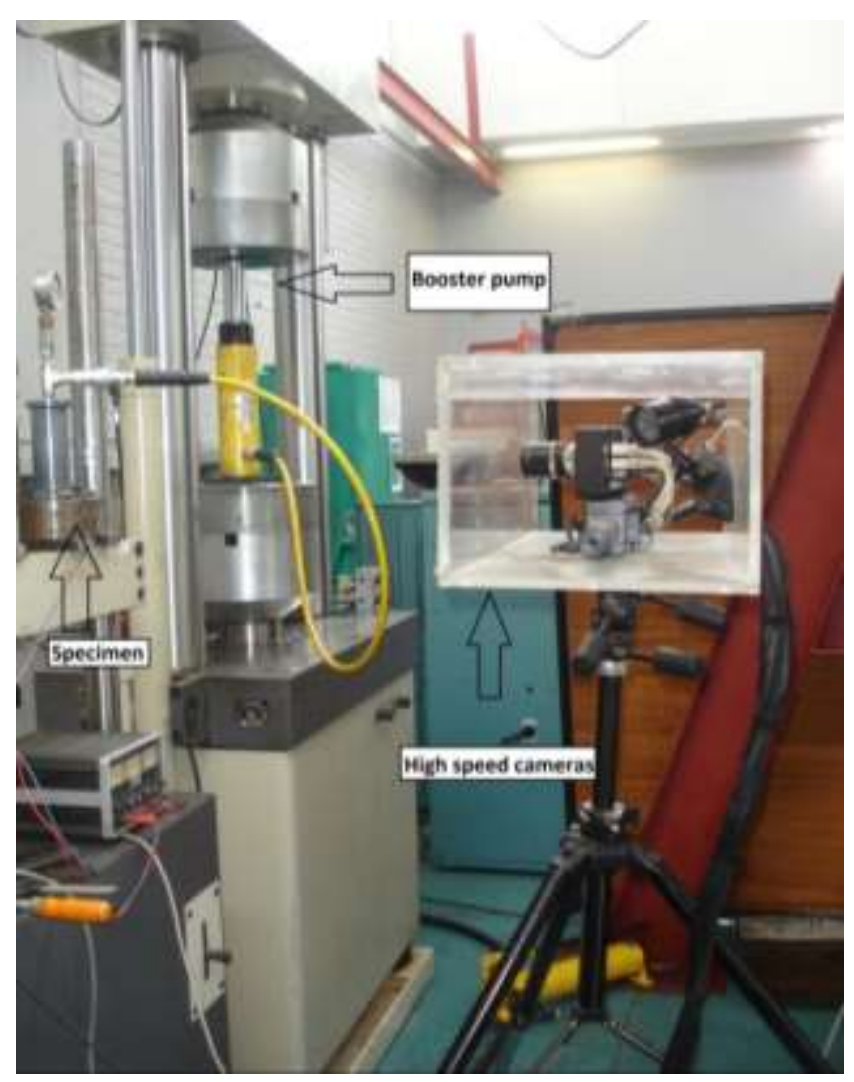

Figure 9. The experimental setup.

\subsubsection{Measurement methods}

Because of the movement of the measuring points, a non-contact optical 3D measuring system was employed. In order to measure displacement at the selected points, two highspeed cameras were used to record the movement of the selected points on the surface of the bulged area (see Figure 9 and Figure 10) with a frame rate of 3 frames per second (the highspeed capability of the camera was not used in the current study). At the end of the test, the results were exported to the GOM PONTOS software (2009) to calculate the displacement of the points. The PONTOS software was used to detect the positions of the marked circles on the surface of the tube.

An analogue pressure transducer (0-60 MPa) was coupled with the GOM to record the applied pressure at the sampling times. 


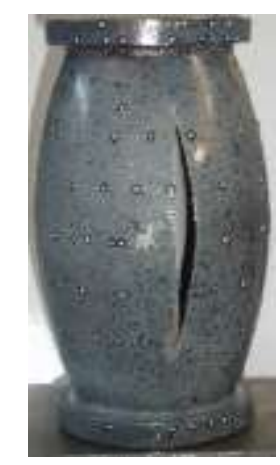

Figure 10. Test sample.

\subsection{FEA of the tube bulge test}

In this study, the isotropic multi-linear hardening model along with Hill48 yield criterion were employed to define the material behaviour. Like the work done by (Zribi et al., 2013), the Lankford anisotropy coefficients were determined using tensile test specimens, flattened and cut in axial and circumferential directions. The strain in length and width of the samples were measured directly, while the thickness strain was inferred assuming noncompressibility of the material. $\mathrm{r}_{0}$ and $\mathrm{r}_{90}$ were determined as 0.87 and 0.82 respectively. The total displacement, comprising circumferential and longitudinal displacements, versus the internal pressure was simulated so that it could be used in the optimization algorithm of the inverse problem. In order to reduce the computational time, an axisymmetric model of the assembled tube, containing the tube and the free-to-move cap, was modelled using the ANSYS implicit software (see Figure 11). Four elements through the thickness and 298 elements along the length of the tube were found sufficient to converge to a result with a maximum displacement error of less than $0.005 \mathrm{~mm}$. It is noteworthy that the mesh dependency analysis was done after obtaining the stress-strain curve, and the whole process was subsequently repeated with the optimum mesh size.

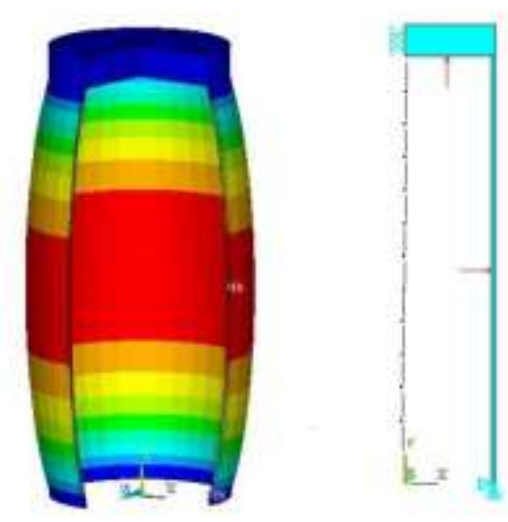

Figure 11. FE model and the boundary conditions. 


\subsection{Surrogate modelling}

A genetic multi-objective optimization algorithm (implemented as "gamultiobj" in Matlab (2015) using the default parameters), was adopted to solve the multi-objective optimization problem. The algorithm calls for multiple evaluations of the objective functions. Running a finite element simulation to evaluate the objective functions for each call, drastically increases the computational cost of the entire of the algorithm. To deal with this, we employed a cheap-to-evaluate surrogate model of the FEM. In other words, the algorithm calls the surrogate model for the objective function evaluation rather than calling the FEM. The surrogate model used in this study is an Artificial Neural Network which was trained to replace the computationally expensive FEM. Here the term training refers to identifying parameters of the regression model (weights of the basis function used in the ANN). In order to train the ANN, it is common practice to construct the set of the material parameters, and the corresponding finite element simulation responses, and then the ANN is trained to estimate the FEM response for the unseen parameter. e.g. see (Aguir et al., 2011). This set is known as the training set.

In order to identify each increment, the ANNs are trained. We simulated the experiment using six different hardening rates covering high and low hardening rates for each increment, assuming an initial large increment size, e.g. see Figure 1. It is better to adjust the simulation pressure high enough to ensure that the optimization algorithm can search for possible large increments. However for the initial increments we set the simulation pressure lower than the maximum obtained pressure in the experiment to reduce the computational costs. Selecting these lower pressures for the simulations does not degrade the accuracy, but the computational cost increases by imposing small increments to the algorithm, while it is not necessary. The hardening rates were used as inputs in the training set.

We did not directly introduce the high dimensional displacement vectors from the simulated responses as the outputs of the training set. Instead, the first principal component (PC) of the simulation response vectors served as the output of the training set to construct the surrogate model. We replaced the original simulation response vectors with the PCs to simplify the ANN architecture. PCs were obtained by projecting the response vectors into the space of the principal directions of the training set. For more information about principal component analysis see (Jolliffe, 2002). This projection technique allows reducing the dimensionality of the vectors, while most of the variance in the data is retained. For instance, using the first principal component for the simulation responses shown in Figure 4 reduced the response dimensions from 15 to 1, while it retained more than $99 \%$ of the variance. Hence, the authors strongly suggest using PCs as substitute for the original vectors in constructing the surrogate model to simplify the structure while maintaining the accuracy.

The constructed training set is then used to train the ANNs. This very simple network contains one neuron for the input layer, two neurons N1 and N2 (see Figure 12) for the hidden layer, and one neuron for the output layer. The selected training algorithm is the Bayesian "trainbr"' training function in Matlab. Figure 12 schematically illustrates 
the procedure of constructing the cheap-to-evaluate surrogate model of the finite element simulation.

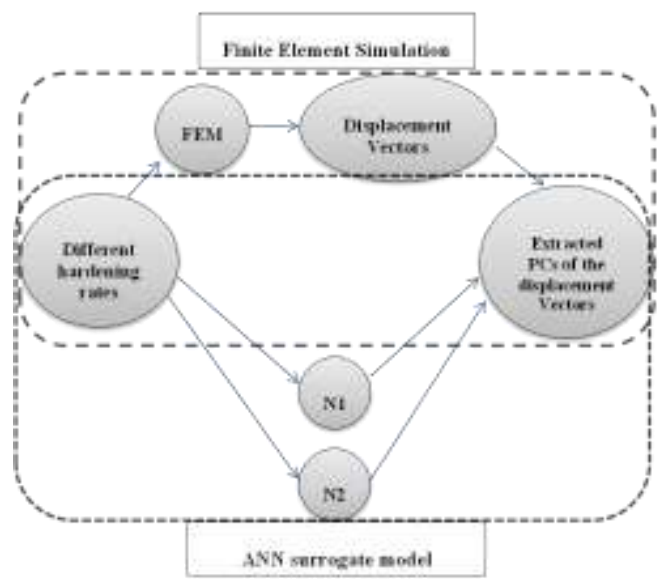

Figure 12. Schematic of constructing the surrogate model.

Figure 13 illustrates the entire of the proposed computationally efficient inverse method, used to estimate stress-strain behaviour of the tube, subjected to tube bulge test. 


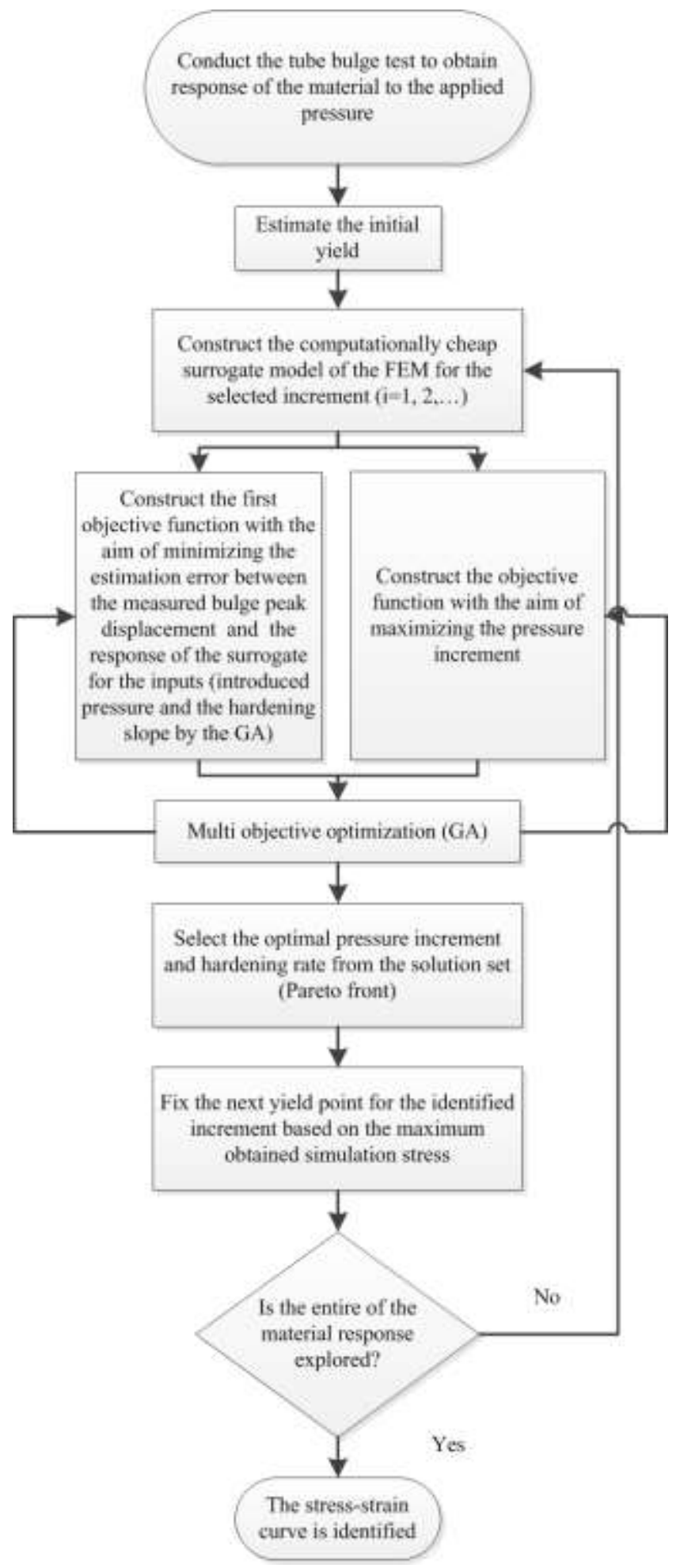

Figure 13. The efficient inverse identification procedure used to identify the stress-strain behaviour of the tube in the tube bulge test. 


\section{Results and discussion}

In order to provide a baseline for evaluating the efficacy of the newly proposed method, a comparative study was done using the conventional forward inverse identification method. This method assumes an a priori hardening model, to capture the stress-strain behaviour of the tube. By way of example, the procedure used in (Zribi et al., 2013) was applied to identify parameters of the Ludwik model for the response of the material obtained from the bulge test. The FEA of the test was employed to simulate the response of the experiment.

In these calculations the simulation pressure was limited to $25.5 \mathrm{MPa}$, although the maximum pressure obtained in the test was $26.4 \mathrm{MPa}$. The characterisation process was therefore restricted to a part of the stress-strain curve associated with the tube bulge test response. This was because the FEA of the material parameters suggested by the optimization algorithm could not be made to converge for many of the material parameters suggested by the optimization algorithm, due to the instability of the numerical model given the material characteristic at the selected pressure. This is more severe when the suggested stress-strain response approaches the stress-strain behaviour of the material. Nonconvergence of the FEA for the suggested parameters can therefore degrade the efficiency of the optimization algorithm.

The best fitted Ludwik model is represented by

$$
267+977 \varepsilon^{0.55}
$$

where $\sigma$ and $\varepsilon$ denote stress and plastic strain. Using this Ludwik model to simulate the process, a simulated response may be found which is plotted on the measured material response in Figure 14.

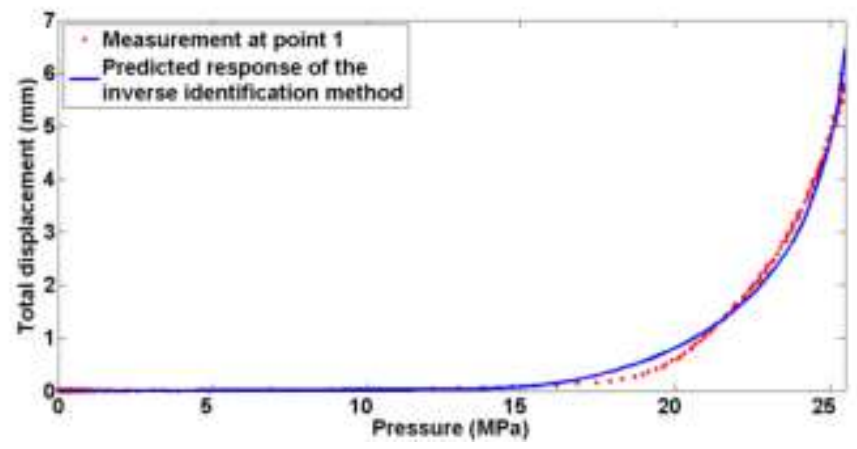

Figure 14. Simulation response of the identified Ludwik model and the measured material response.

The Ludwik material model is clearly not able to accurately predict the material response for the selected part of the experiment. Other material hardening models could also be tried, and the optimization algorithm could be restarted at different initial points to result in possibly a more accurate stress-strain curve. This example was the first attempt of the authors to obtain the stress-strain behaviour of the selected tube. However, the inaccurate result, and high computational expense of each trial, restarting the optimization algorithm and selecting 
different hardening models, motivated us to opt for a new inverse identification approach as an alternative to the conventional inverse problem. We here employ the progressive inverse stress-strain identification approach to estimate the material hardening behaviour.

In order to estimate the stress-strain characteristic of the tube, we limited the acceptable estimation error for determining parameters of the first three increments to $4 \times 10^{-4} \mathrm{~mm}^{2}$. This error is the average of the squared differences of the measured displacements and the simulated displacements of the measured points within each increment. In addition, the acceptable estimation error for the rest of the increments is limited to $2 \times 10^{-2}$ (dimensionless) as the average of the squared relative error of the simulated and measured displacement within each increment, as it is used in (Zribi et al., 2013, Aguir et al., 2011). We used absolute error for the first three increments because the magnitude of some measurements in the beginning of the response measurement is very small while their measurement noise is of the same order as the values. Therefore, dividing the differences by a very small number for calculating the relative error imposes a dominant error for calculating the average error within the increment. This problem is mostly due to the fact that we use the same devices for measuring very low and very high magnitudes during the test. The objective of this paper is however not to study different types of error and variations of the error along the experiment. More research could be conducted to assess the effects of the different objective functions.

Applying the progressive inverse material identification method to the response of the bulge test, the stress-strain behaviour of the material is identified. As it is illustrated in Figure 15 the algorithm estimates the hardening behaviour with 7 linear increments.

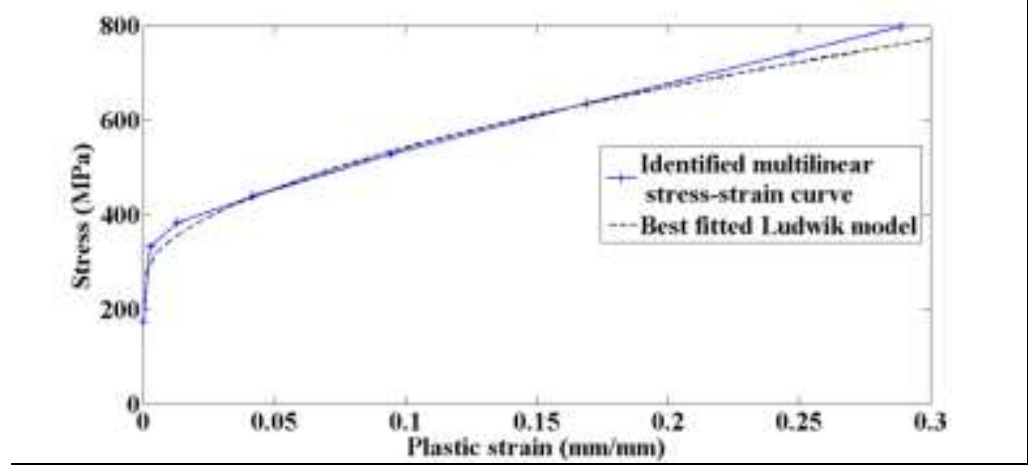

Figure 15. Identified multilinear stress-strain curve against the best fitted Ludwik model.

In order to evaluate the accuracy of the stress-strain behaviour obtained, the identified curve is used to simulate the experiment. Responses of the simulation and responses of the experiment are compared in Figure 16. Measurements at point 1 (see Figure 8) were employed in the progressive inverse identification method. The responses of the tube at points 2 and 3 were not used in the calculations. They could therefore be used to validate the result. Clearly, using the obtained stress-strain behaviour in the simulation process allows the simulations to accurately follow the material responses at the selected points. 


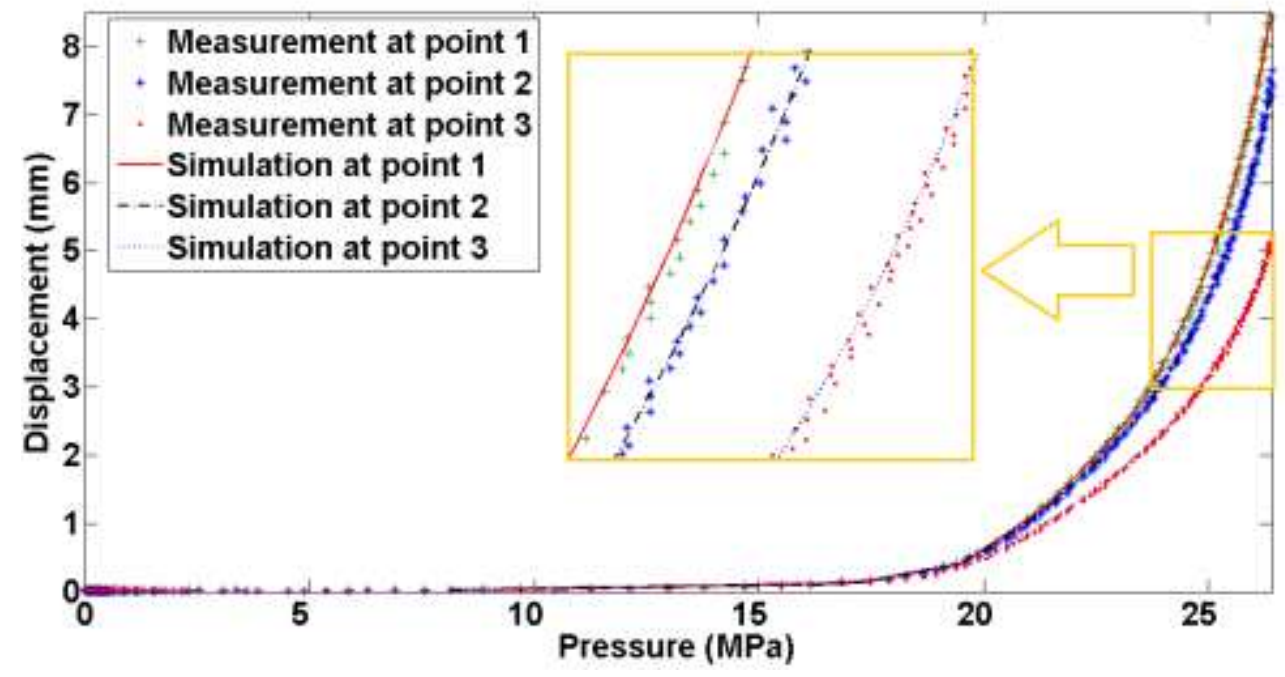

Figure 16. Response of the tube bulge simulation using the obtained material behaviour.

\section{Conclusion}

This study proposed an inverse identification approach to identify the flow stress of the materials. The proposed approach estimates the stress-strain behaviour with a multi-linear curve with adjustable resolution. In addition, a procedure to reduce the total computational cost was explained. The proposed method retains the benefits of the both analytical and conventional inverse flow stress identification approaches. On the one hand the method is independent of selecting an a priori constitutive hardening model, and on the other hand various simplifying assumptions and difficult measurements are avoided.

The presented method was employed to identify stress-strain behaviour of a 304 stainless steel tube in a tube bulge test. The comparison between response of the simulation, using the obtained material behaviour, and the response of the material showed the efficiency of the method.

As a result, the progressive inverse identification method can be employed efficiently to identify the stress-strain behaviour of the materials with an unknown hardening constitutive model subjected to complicated stress states, within a reasonable time. This is possible owing to the changes proposed to the conventional inverse forward identification method.

\section{References}

ANSYS® Academic Research,Release 14,5.

2009. GOM Optical Measuring Techniques. PONTOS User Manual - Software. PONTOS. 6.2 ed. 
2015. Matlab. R2015 ed. Natick, Massachusetts: The Mathworks Inc.

AGUIR, H., BELHADJSALAH, H. \& HAMBLI, R. (2011), "Parameter identification of an elastoplastic behaviour using artificial neural networks-genetic algorithm method", Materials and Design, 32, 48-53.

BOCCIARELlI, M., BULJAK, V., MOY, C. K. S., RINGER, S. P. \& RANZI, G. (2014), "An inverse analysis approach based on a POD direct model for the mechanical characterization of metallic materials", Computational Materials Science, 95, 302-308.

BOLZON, G. \& TALASSI, M. (2013), "An effective inverse analysis tool for parameter identification of anisotropic material models", International Journal of Mechanical Sciences, 77, 130-144.

BORTOT, P., CERETTI, E. \& GIARDINI, C. (2008), "The determination of flow stress of tubular material for hydroforming applications", Journal of Materials Processing Technology, 203, 381-388.

COONEY, G. M., MOERMAN, K. M., TAKAZA, M., WINTER, D. C. \& SIMMS, C. K. (2015), "Uniaxial and biaxial mechanical properties of porcine linea alba", Journal of the Mechanical Behavior of Biomedical Materials, 41, 68-82.

DEB, K. \& KALYANMOY, D. (2001). Multi-Objective Optimization Using Evolutionary Algorithms, John Wiley \& Sons, Inc.

DUREJA, A. K., SINHA, S. K., PAWASKAR, D. N., SESHU, P., CHAKRAVARTTY, J. K. \& SINHA, R. K. (2014), "Modelling flow and work hardening behaviour of cold worked Zr$2.5 \mathrm{Nb}$ pressure tube material in the temperature range of $30-600 ;{ }^{\circ} \mathrm{C}$, Nuclear Engineering and Design, 269, 52-56.

GERMAIN, G., MOREL, A. \& BRAHAM-BOUCHNAK, T. (2013), "Identification of material constitutive laws representative of machining conditions for two titanium alloys: Ti6Al4V and Ti555-3", Journal of Engineering Materials and Technology, Transactions of the ASME, 135 .

HWANG, Y. M., LIN, Y. K. \& ALTAN, T. (2007), "Evaluation of tubular materials by a hydraulic bulge test", International Journal of Machine Tools and Manufacture, 47, 343-351.

HWANG, Y. M. \& WANG, C. W. (2009), "Flow stress evaluation of zinc copper and carbon steel tubes by hydraulic bulge tests considering their anisotropy", Journal of Materials Processing Technology, 209, 4423-4428.

JOLLIFFE, I. T. (2002). Principal component analysis, Springer-Verlag.

KANG, S.-H., LEE, Y.-S. \& LEE, H. W. (2014), "Evaluation of flow stress and damage index at large plastic strain by simulating tensile test of Al6061 plates with various grain sizes", International Journal of Mechanical Sciences, 80, 54-61.

KOÇ, M., AUE-U-LAN, Y. \& ALTAN, T. (2001), "On the characteristics of tubular materials for hydroforming - experimentation and analysis", International Journal of Machine Tools and Manufacture, 41, 761-772.

LI, G., XU, F., SUN, G. \& LI, Q. (2014), "Identification of mechanical properties of the weld line by combining 3D digital image correlation with inverse modeling procedure", International Journal of Advanced Manufacturing Technology, 74, 893-905. 
LIANFA, Y. \& CHENG, G. (2008), "Determination of stress-strain relationship of tubular material with hydraulic bulge test", Thin-Walled Structures, 46, 147-154.

LUCZYNSKI, K. W., STEIGER-THIRSFELD, A., BERNARDI, J., EBERHARDSTEINER, J. \& HELLMICH, C. (2015), "Extracellular bone matrix exhibits hardening elastoplasticity and more than double cortical strength: Evidence from homogeneous compression of non-tapered single micron-sized pillars welded to a rigid substrate", Journal of the Mechanical Behavior of Biomedical Materials, 52, 51-62.

NEMAT-ALLA, M. (2003), "Reproducing hoop stress-strain behavior for tubular material using lateral compression test", International Journal of Mechanical Sciences, 45, 605-621.

NGUYEN, N. T., LEE, M. G., KIM, J. H. \& KIM, H. Y. (2013), "A practical constitutive model for AZ31B Mg alloy sheets with unusual stress-strain response", Finite Elements in Analysis and Design, 76, 39-49.

NOH, W., KOH, Y., SEOK, D.-Y., WAGONER, R. H., BARLAT, F. \& CHUNG, K. (2014), "Mechanical property of magnesium alloy sheet with hardening deterioration at warm temperatures and its application for failure analysis: Part I - property characterization", International Journal of Material Forming, 1-9.

PRATES, P. A., OLIVEIRA, M. C. \& FERNANDES, J. V. (2014), "A new strategy for the simultaneous identification of constitutive laws parameters of metal sheets using a single test", Computational Materials Science, 85, 102-120.

PRATES, P. A., OLIVEIRA, M. C. \& FERNANDES, J. V. (2015), "Identification of material parameters for thin sheets from single biaxial tensile test using a sequential inverse identification strategy", International Journal of Material Forming, 1-25.

SABOORI, M., CHAMPLIAUD, H., GHOLIPOUR, J., GAKWAYA, A., SAVOIE, J. \& WANJARA, P. (2014), "Evaluating the flow stress of aerospace alloys for tube hydroforming process by free expansion testing", International Journal of Advanced Manufacturing Technology, 72, 1275-1286.

STRANO, M. \& ALTAN, T. (2004), "An inverse energy approach to determine the flow stress of tubular materials for hydroforming applications", Journal of Materials Processing Technology, 146, 92-96.

SUN, G., XU, F., LI, G., HUANG, X. \& LI, Q. (2014), "Determination of mechanical properties of the weld line by combining micro-indentation with inverse modeling", Computational Materials Science, 85, 347-362.

XU, Y., CHAN, L. C., TSIEN, Y. C., GAO, L. \& ZHENG, P. F. (2008), "Prediction of workhardening coefficient and exponential by adaptive inverse finite element method for tubular material", Journal of Materials Processing Technology, 201, 413-418.

ZHANG, S., LEOTOING, L., GUINES, D., THUILLIER, S. \& ZANG, S.-L. (2014), "Calibration of anisotropic yield criterion with conventional tests or biaxial test", International Journal of Mechanical Sciences, 85, 142-151.

ZRIBI, T., KHALFALLAH, A. \& BELHADJSALAH, H. (2013), "Experimental characterization and inverse constitutive parameters identification of tubular materials for tube hydroforming process", Materials \& Design, 49, 866-877. 